JOURNAL OF

SYNCHROTRON

RADIATION
Received 11 June 2016

Accepted 15 June 2016

Edited by A. Momose, Tohoku University, Japan

Keywords: nonlinear diffraction; Bragg case; reflection region; third-order nonlinearity; two-wave diffraction

\section{X-ray third-order nonlinear plane-wave Bragg-case dynamical diffraction effects in a perfect crystal. Erratum}

\author{
Minas K. Balyan*
}

Faculty of Physics, Department of Solid State Physics, Yerevan State University, Alex Manoogian 1, Yerevan 0025, Armenia. *Correspondence e-mail: mbalyan@ysu.am

Formulae in the paper by Balyan (2015) [J. Synchrotron Rad. 22, 1410-1418] are corrected.

The formulae (2) and (4) in the paper Balyan (2015) have the same essential typographical error. The correct forms of these equations are

$$
\operatorname{rot} \operatorname{rot} \tilde{\tilde{\mathbf{E}}}+\frac{1}{c^{2}} \frac{\partial^{2}}{\partial t^{2}}=-\frac{1}{\varepsilon_{0} c^{2}} \frac{\partial^{2}}{\partial t^{2}}
$$

and

$$
\operatorname{rot} \operatorname{rot} \tilde{\mathbf{E}}\left(\mathbf{r}, \omega_{q}\right)-\frac{\omega_{q}^{2}}{c^{2}}\left[1+\chi^{(1)}\left(\mathbf{r}, \omega_{q}\right)\right] \tilde{\mathbf{E}}\left(\mathbf{r}, \omega_{q}\right)=\frac{\omega_{q}^{2}}{\varepsilon_{0} c^{2}} \tilde{\mathbf{P}}^{\mathrm{NL}}\left(\mathbf{r}, \omega_{q}\right) \text {. }
$$

In the same paper, the formulae (17) and (19) are also incorrect. The correct forms of these equations are

$$
E^{(i)}(x, 0)=E_{0}^{(i)} \exp \left(i k \cos \theta^{(i)} x\right)
$$

and

$$
\begin{aligned}
E_{0}(x, 0) & =E_{0}^{(i)} \exp (-i k \sin \theta \Delta \theta x), \\
E_{h}(x, T) & =0
\end{aligned}
$$

These equations are used for derivation of the third-order nonlinear Takagi's equations.

\section{References}

Balyan, M. K. (2015). J. Synchrotron Rad. 22, 1410-1418.

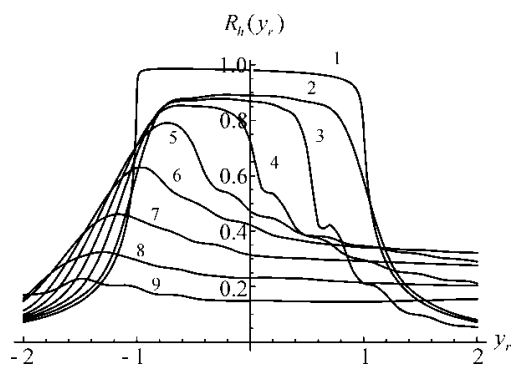

(C) 2016 International Union of Crystallography 